\title{
RANDOM CLOSED SETS VIEWED AS RANDOM RECURSIONS
}

\author{
R. DANIEL MAULDIN AND ALEXANDER P. MCLINDEN
}

\begin{abstract}
It is known that the box dimension of any Martin-Löf random closed set of $\{0,1\}^{\mathbb{N}}$ is $\log _{2}\left(\frac{4}{3}\right)$. Barmpalias et al. [Journal of Logic and Computation, Vol. 17, No. 6 (2007)] gave one method of producing such random closed sets and then computed the box dimension, and posed several questions regarding other methods of construction. We outline a method using random recursive constructions for computing the Hausdorff dimension of almost every random closed set of $\{0,1\}^{\mathbb{N}}$, and propose a general method for random closed sets in other spaces. We further find both the appropriate dimensional Hausdorff measure and the exact Hausdorff dimension for such random closed sets.
\end{abstract}

\section{INTRODUCTION}

In [1], a specific method of producing random closed sets of $\{0,1\}^{\mathbb{N}}$ is proposed and the box dimension of every Martin-Löf random closed set is shown to be $\eta=$ $\log _{2}\left(\frac{4}{3}\right)$. Our goal will be to outline a method from [6] for computing Hausdorff dimension and Hausdorff measure in that dimension, and moreover a method to find the exact Hausdorff dimension functions for random recursions from [5], and to show how this method can be used to compute the Hausdorff dimension and exact Hausdorff dimension of almost every random closed set. More specifically, our main goal will be to prove the following theorem:

Theorem 1. For almost every random closed set $Q, \operatorname{dim}_{H}(Q)=\eta$. Moreover, $\mathcal{H}^{\eta}(Q)=0$ almost surely. Let $h(t)=t^{\eta} \log (|\log (t)|)^{2-\frac{\log (4)}{\log (3)}}$. Then, for almost every $Q, 0<\mathcal{H}^{h}(Q)<\infty$.

First we will outline a definition for random closed sets. Note that this differs from the definition given in [1], for two reasons. Firstly, Barmpalias et al. were concerned primarily with Martin-Löf randomness, whereas we will construct our closed sets based on probability. Secondly, we will not be concerned about coding a construction which is not one-to-one (i.e. we may have multiple codes for a single set). If $S$ is a set, define $S^{*}$ as the set of all finite sequences from $S$, including the empty sequence $\emptyset$. If $\alpha=\left(a_{1}, \ldots, a_{n}\right) \in S^{*}$, and $\beta=\left(b_{1}, \ldots, b_{m}\right) \in S^{*}$, then let $|\alpha|=n$ and $\alpha * \beta=\left(a_{1}, \ldots, a_{n}, b_{1}, \ldots, b_{m}\right)$. The general setting for a random closed set construction is $\{0,1\}^{\mathbb{N}}$, with the ultrametric $\rho(\sigma, \tau)=\frac{1}{2^{|\sigma \wedge \tau|+1}}$, where for distinct $\sigma, \tau \in\{0,1\}^{\mathbb{N}}, \sigma \wedge \tau$ is the longest finite string which agrees with both of $\tau$ and $\sigma$. We have a probability space $(\Omega, \Sigma, P)$, and a collection of $Q_{\sigma}$ indexed by $\sigma \in\{0,1\}^{*}$, so that

1991 Mathematics Subject Classification. Primary 60D05; Secondary 60B05.

Key words and phrases. Random closed sets, Random recursion.

Both authors were supported in part by NSF grants DMS 0700831 and DMS 0652450. 
(1) each $Q_{\sigma}: \Omega \rightarrow \mathcal{K}\left(\{0,1\}^{\mathbb{N}}\right)$, where $\mathcal{K}\left(\{0,1\}^{\mathbb{N}}\right)$ denotes the space of compact subsets of $\{0,1\}^{\mathbb{N}}$

(2) $Q_{\emptyset}=\{0,1\}^{\mathbb{N}}$ with probability 1

(3) for each $\sigma \in\{0,1\}^{*}, P\left(Q_{\sigma * 0}=[\sigma * 0], Q_{\sigma * 1}=[\sigma * 1] \mid Q_{\sigma} \neq \emptyset\right)=P\left(Q_{\sigma * 0}=\right.$ $\left.[\sigma * 0], Q_{\sigma * 1}=\emptyset \mid Q_{\sigma} \neq \emptyset\right)=P\left(Q_{\sigma * 0}=\emptyset, Q_{\sigma * 1}=[\sigma * 1] \mid Q_{\sigma} \neq \emptyset\right)=\frac{1}{3}$, where $[\tau]$ is the set of words in $\{0,1\}^{\mathbb{N}}$ which agree with $\tau$ for the entire length of $\tau$. set.

Define $Q(\omega)=\bigcap_{n \geq 0} \bigcup_{|\sigma|=n} Q_{\sigma}(\omega)$. For each $\omega \in \Omega, Q(\omega)$ is called a random closed

In comparison, the general model from [6] is as follows.

Fix a Euclidean space $\mathbb{R}^{m}$, and a nonempty compact subset $J \subset \mathbb{R}^{m}$, so that $\operatorname{cl}(\operatorname{int}(J))=J$. Assume that we have a probability space $(\Omega, \Sigma, P)$ and a family of random subsets of $\mathbb{R}^{m}$,

satisfying three properties:

$$
\mathbf{J}=\left\{\mathbf{J}_{\sigma} \mid \sigma \in \mathbb{N}^{*}\right\}
$$

(1) $J_{\emptyset}(\omega)=J$ for almost all $\omega \in \Omega$. For every $\sigma \in \mathbb{N}^{*}$ and for almost all $\omega$, if $J_{\sigma}(\omega)$ is nonempty, then $J_{\sigma}(\omega)$ is geometrically similar to $J$.

(2) For almost every $\omega$ and for every $\sigma \in \mathbb{N}^{*}, J_{\sigma * 1}(\omega), J_{\sigma * 2}(\omega), \ldots$ is a sequence of nonoverlapping subsets of $J_{\sigma}(\omega)$. (By " $A$ and $B$ are nonoverlapping" we mean that int $A \cap \operatorname{int} B=\emptyset$.)

(3) The random vectors $\tau_{\sigma}=\left\langle T_{\sigma * 1}, T_{\sigma * 2}, \ldots\right\rangle, \sigma \in \mathbb{N}^{*}$, are i.i.d., where $T_{\sigma * n}(\omega)$ is the ratio of the diameter of $J_{\sigma * n}(\omega)$ to the diameter of $J_{\sigma}(\omega)$ if $J_{\sigma}(\omega)$ is nonempty. (For convenience, let $T_{\emptyset}(\omega)=$ diameter of $J$.)

We call such a system $\mathbf{J}$ a construction. Our constructions require only a "stochastic ratio self similarity", and also allow for infinite branching. We now define the random set $K$ by

$$
K(\omega)=\bigcap_{n \geq 1} \bigcup_{\sigma \in \mathbb{N}^{n}} J_{\sigma}(\omega) .
$$

For convenience, let $0^{0}=0$. Then $\sum_{p \geq 1} T_{\sigma * p}^{0}(\omega)$ counts the number of nonempty $J_{\sigma * p}(\omega)$, if $J_{\sigma}(\omega)$ is itself nonempty. For $\beta \geq 0$, define $\Phi:[0, \infty) \rightarrow[0, \infty)$ by $\Phi(\beta)=E\left(\sum_{n \geq 1} T_{n}^{\beta}\right)=E\left(\sum_{n \geq 1} T_{\sigma * n}^{\beta}\right)$. Note that the assumption " $\Phi(0)>1 "$, well known from branching processes, as explained in [6], guarantees that with positive probability, $K \neq \emptyset$.

The following theorems are some results from random recursive constructions that we will use to examine random closed sets. In these theorems we use the following notation: if $(X, \rho)$ is a metric space, and $E \subset X$, then $\operatorname{dim}_{H, \rho}(E)$ denotes 
the Hausdorff dimension of $E$ with respect to the $\rho$ metric (For a reference on Hausdorff dimension/measure, try [7] or [3]. For a reference on exact Hausdorff dimension/measure, try [8]). Similarly, $\mathcal{H}_{\rho}^{\alpha}$ is the dimension $\alpha$ Hausdorff measure with respect to $\rho$, and $\mathcal{H}_{\rho}^{g}$ is the Hausdorff measure with respect to the gauge function $g$ and metric $\rho$. In the case where $\rho$ is understood, we omit the subscript $\rho$.

Theorem 2. Suppose $\Phi(0)>1$. Then, with positive probability, $K$ is nonempty. Moreover, if $\alpha$ is the least $\beta$ so that $\Phi(\beta) \leq 1$, then if $K \neq \emptyset, \alpha$ is almost surely the Hausdorff dimension of $K$; i.e. $P\left(\operatorname{dim}_{H}(K)=\alpha \mid K \neq \emptyset\right)=1$.

The proof of this, just as the setup outlined above, is contained in [6].

In the case $|S|=n<\infty$, the construction is called an $n$-ary construction. In this case, much more is known. We give two examples of what is known which can be applied to the main model given in [1].

Theorem 3. With $\alpha$ defined as in Theorem 2, if $P\left(\sum_{1 \leq i \leq n} T_{i}^{\alpha} \neq 1\right)>0$, then $\mathcal{H}^{\alpha}(K)=0$ almost surely.

This is Theorem 7.7 from [4].

Theorem 4. Suppose that $\mathbf{J}$ is an n-ary construction with $\Phi(0)>1$, and suppose that each $T_{i}$ takes only finitely many values $P-$ a.e. and that $P\left(\sum_{1 \leq i \leq n} T_{i}^{\alpha} \neq 1\right)>0$. Then, for $P-$ a.e. $\omega$, if $K(\omega) \neq \emptyset$, then

where $h(t)=t^{\alpha}(\log |\log (t)|)^{1-\frac{\alpha}{m}}$.

$$
0<\mathcal{H}^{h}(K(\omega))<\infty,
$$

This is only a specific case using Theorems $5.1-5.4$ from [5].

Next is a lemma which will be necessary to convert from random closed sets to random recursions. It is primarily concerned with how Hausdorff dimension and measure change under Hölder (and hence bi-Hölder) maps.

Lemma 5. Let $(X, \rho),(Y, r)$ be metric spaces, and $\phi: X \rightarrow Y$, with $\phi$ Hölder of order $d$. That is, for some $m, r(\phi(x), \phi(y)) \leq m \cdot \rho(x, z)^{d}$ for all $x, y \in X$. Then, for $\beta \geq 0, \mathcal{H}_{r}^{\beta}(\phi(E)) \leq m^{\beta} \mathcal{H}_{\rho}^{d \beta}(E)$ for each $E \subset X$. Moreover, if $E \subset X$, then $\operatorname{dim}_{H, \rho}(E) \geq d \cdot \operatorname{dim}_{H, r}(\phi(E))$.

Proof. Let $E \subset X$. If $G$ is a $\delta$-mesh cover of $\mathrm{E}$ (meaning each set in $G$ has diameter $\leq \delta)$, then if $A \in G, \operatorname{diam}(\phi(A)) \leq m \delta^{d}$, so $\phi(G):=\{\phi(A): A \in G\}$ is an $m \delta^{d}$-mesh cover of $\phi(E)$. Thus,

$$
\begin{aligned}
& \mathcal{H}_{m \delta^{d}, r}^{\beta}(\phi(E))=\inf \left\{\sum_{I \in \mathcal{J}}|I|^{\beta}: \mathcal{J} \text { is an } m \delta^{\beta} \text {-mesh cover of } \phi(E)\right\} \\
& \leq \inf \left\{\sum_{A \in G}|\phi(A)|^{\beta}: A \in G, G \text { is a } \delta \text {-mesh cover of } E\right\} \\
& \leq \inf \left\{\sum_{A \in G} m^{\beta}|A|^{d \beta}: A \in G, G \text { is a } \delta \text {-mesh cover of } E\right\} \\
& =m^{\beta} \cdot \inf \left\{\sum_{A \in G}|A|^{d \beta}: A \in G, G \text { is a } \delta \text {-mesh cover of } E\right\}
\end{aligned}
$$


$=m^{\beta} \mathcal{H}_{\delta, \rho}^{d \beta}(E)$.

Letting $\delta \rightarrow 0, \mathcal{H}_{r}^{\beta}(\phi(E)) \leq m^{\beta} \mathcal{H}_{\rho}^{d \beta}(E)$. Also, note that if $\mathcal{H}_{r}^{\beta}(\phi(E))=\infty$, then $\mathcal{H}_{\rho}^{d \beta}(E)=\infty$, and hence $\operatorname{dim}_{H, \rho}(E) \geq d \cdot \operatorname{dim}_{H, r}(\phi(E))$.

Note that in the case $\phi$ is bi-Hölder of order $d$, i.e. $m_{1} \cdot \rho(x, z)^{d} \leq r(\phi(x), \phi(z)) \leq$ $m_{2} \cdot \rho(x, z)^{d}$, for some $m_{1}, m_{2}>0$, then there is equality in the last statement of Lemma 5 .

\section{Random Closed Sets Without Dying}

We say the construction outlined from [1] is "without dying", since there is 0 probability that a node in the tree has no children. In order to compute the almostsure Hausdorff dimension of these random closed sets, we will transfer the problem to a random recursive construction and then use Lemma 5 to relate the two. In order to do this, we will map each $Q$ to a random subset of Cantor's middle third set (call it $\mathcal{C}$ ), and we will find the Hausdorff dimension with respect to the standard Euclidean metric. If $\phi:\{0,1\}^{\mathbb{N}} \rightarrow \mathcal{C}$ is defined by $\phi(\sigma)=\sum_{i \geq 1} \frac{2 \sigma(i)}{3^{i}}$, then $\phi$ is a bijection. Translating (1)-(3) from the above description of random closed sets, define $J_{\emptyset}(\omega)=[0,1]$, then for each $\sigma \in\{0,1\}^{*}$, if $J_{\sigma}(\omega) \neq \emptyset$, define $J_{\sigma * 0}(\omega)$ as the left third of the interval $J_{\sigma}(\omega)$ if $Q_{\sigma * 0}(\omega) \neq \emptyset$ and $J_{\sigma * 0}(\omega)=\emptyset$ otherwise. Similarly define $J_{\sigma * 1}(\omega)$ as the right third. This is simply a random recursive construction, where the reduction ratios are, with equal probability, one of three things: $T_{\sigma * 0}=T_{\sigma * 1}=\frac{1}{3}, T_{\sigma * 0}=0$ and $T_{\sigma * 1}=\frac{1}{3}$, or $T_{\sigma * 0}=\frac{1}{3}$ and $T_{\sigma * 1}=0$. Also, by construction, $\phi(Q(\omega))=K(\omega)$ almost surely.

Then, if $\beta \geq 0, \Phi(\beta)=E\left(T_{0}^{\beta}+T_{1}^{\beta}\right)=2 \frac{2}{3}\left(\frac{1}{3}\right)^{\beta}$, which is 1 only for $\beta=\frac{\log (4)}{\log (3)}-1$, so by Theorem 2, the Hausdorff dimension of $K$ is almost surely $\alpha=\frac{\log (4)}{\log (3)}-1$.

Also notice that if $\sigma, \tau \in\{0,1\}^{\mathbb{N}}$, then

$$
\rho(\sigma, \tau)^{d} \leq|\phi(\sigma)-\phi(\tau)| \leq 3 \rho(\sigma, \tau)^{d},
$$

where $d=\frac{\log 3}{\log 2}$, since

$$
\begin{gathered}
|\phi(\sigma)-\phi(\tau)|=\left|\sum_{i \geq 1} \frac{2(\sigma(i)-\tau(i))}{3^{i}}\right|=\left|\sum_{i>|\sigma \wedge \tau|} \frac{2(\sigma(i)-\tau(i))}{3^{i}}\right| \\
\leq \sum_{i>|\sigma \wedge \tau|} \frac{2}{3^{i}}=\frac{1}{3^{|\sigma \wedge \tau|}}=3 \rho(\sigma, \tau)^{d}
\end{gathered}
$$

and

$$
\begin{gathered}
|\phi(\sigma)-\phi(\tau)|=\left|\sum_{i>|\sigma \wedge \tau|} \frac{2(\sigma(i)-\tau(i))}{3^{i}}\right| \geq \\
\frac{2}{3^{|\sigma \wedge \tau|+1}}-\sum_{i>|\sigma \wedge \tau|+1} \frac{2}{3^{i}}=\frac{2}{3^{|\sigma \wedge \tau|+1}}-\frac{1}{3^{|\sigma \wedge \tau|+1}}=\rho(\sigma, \tau)^{d} .
\end{gathered}
$$

By 5 and the remarks that follow it, this bi-Hölder inequality implies that, with regard to the $\rho$ metric, the Hausdorff dimension of $Q$ is almost surely $d \cdot \alpha=$ $\left(\frac{\ln 4}{\ln 3}-1\right)\left(\frac{\ln 3}{\ln 2}\right)=\log _{2}\left(\frac{4}{3}\right)=\eta$. Moreover, Theorem 3 gives us that $\mathcal{H}^{\alpha}(K)=0$ almost surely, and so Lemma 5 implies that $\mathcal{H}^{\eta}(Q)=0$ almost surely. 
Note that similar results can be found for packing dimension and upper box dimension by the results contained in [2]. Specifically, one could use Theorem 3.1, which says that if $K \neq \emptyset$, $\operatorname{dim}_{P}(K)=\overline{\operatorname{dim}}_{B}(K)=\operatorname{dim}_{H}(K)=\alpha$ almost surely.

Moreover, we can find the almost-sure exact Hausdorff dimension for $Q$, but first we need the following extension of Lemma 5 .

Lemma 6. Let $(X, \rho),(Y, r)$ be metric spaces, and $\phi: X \rightarrow Y$, with $\phi$ bi-Hölder of order d. That is, there are constants $m_{1}, m_{2}>0$ so that $m_{1} \rho(x, z)^{d} \leq r(\phi(x), \phi(z)) \leq$ $m_{2} \rho(x, z)^{d}$. Let $g(t)=t^{\alpha} \log (|\log (t)|)^{p}$, and $h(t)=t^{\alpha d} \log (|\log (t)|)^{p}$, where $p>0$. Then, if $E \subset X$ so that $0<\mathcal{H}_{r}^{g}(\phi(E))<\infty$, then $0<\mathcal{H}_{\rho}^{h}(E)<\infty$.

The proof of this is similar to the proof of Lemma 5 , using the small additional fact that $\lim _{\delta \rightarrow 0^{+}} \frac{\log \left(\left|\log \left(m \delta^{d}\right)\right|\right)^{p}}{\log (|\log (\delta)|)^{p}}=1$.

Now, to complete the proof of Theorem 1 , we set $h(t)=t^{\eta} \log (|\log (t)|)^{1-\alpha}=$ $t^{\log _{2}\left(\frac{4}{3}\right)} \log (|\log (t)|)^{2-\frac{\log (4)}{\log (3)}}$ and combine Lemmas 4 and 6 , to show that if $Q$ is a random closed set in the above construction, then $P\left(0<\mathcal{H}_{\rho}^{h}(Q)<\infty\right)=1$.

\section{Random Closed Sets of $\{0,1\}^{\mathbb{N}}$ With Dying}

In [1], a question is proposed, where, instead of a random closed set being defined by taking equal probabilities for each node to have both a left and right child, just a left child, or just a right child, we also allow them to have no children. Moreover, we might allow different probabilities for each of "splitting", "keeping the left", "keeping the right", or "dying". That is, when (3) from above is changed to

(3) for each $\sigma \in\{0,1\}^{*}, P\left(Q_{\sigma * 0}=[\sigma * 0], Q_{\sigma * 1}=[\sigma * 1] \mid Q_{\sigma} \neq \emptyset\right)=p_{\text {both }}$, $P\left(Q_{\sigma * 0}=[\sigma * 0], Q_{\sigma * 1}=\emptyset \mid Q_{\sigma} \neq \emptyset\right)=p_{\text {left }}, P\left(Q_{\sigma * 0}=\emptyset, Q_{\sigma * 1}=[\sigma * 1] \mid Q_{\sigma} \neq \emptyset\right)=$ $p_{\text {right }}$, and $P\left(Q_{\sigma * 0}=\emptyset, Q_{\sigma * 1}=\emptyset \mid Q_{\sigma} \neq \emptyset\right)=p_{\text {die }}$.

Theorem 7. For the model of constructing random closed sets of $\{0,1\}^{\mathbb{N}}$ just described, the following hold, if $p_{\text {both }}>p_{\text {die }}$ :

(i) $P(Q \neq \emptyset)>0$

(ii) $P\left(\operatorname{dim}_{H}(Q)=\log _{2}\left(1+p_{\text {both }}-p_{\text {die }}\right) \mid Q \neq \emptyset\right)=1$

(iii) $\mathcal{H}^{\log _{2}\left(1+p_{\text {both }}-p_{\text {die }}\right)}(Q)=0$ almost surely

(iv) If $Q \neq \emptyset$, then $0<\mathcal{H}^{h}(Q)<\infty$ almost surely, where

$$
h(t)=t^{\log _{2}\left(1+p_{\text {both }}-p_{\text {die }}\right)}\left(\log (|\log (t)|)^{1-\log _{3}\left(1+p_{\text {both }}-p_{\text {die }}\right)}\right.
$$

Proof. In this case, we again view this as a random subset of $\mathcal{C}$, in the following manner: $J_{\emptyset}(\omega)=[0,1]$, and for each $\sigma \in\{0,1\}^{*}$, if $J_{\sigma}(\omega) \neq \emptyset$, define $J_{\sigma * 0}(\omega)$ as the left third of the interval $J_{\sigma}(\omega)$ if $Q_{\sigma * 0}(\omega) \neq \emptyset$ and $J_{\sigma * 0}(\omega)=\emptyset$ otherwise. Similarly define $J_{\sigma * 1}(\omega)$ as the right third. In this case, the reductions ratios are, one of four things: $T_{\sigma * 0}=T_{\sigma * 1}=\frac{1}{3}, T_{\sigma * 0}=0$ and $T_{\sigma * 1}=\frac{1}{3}, T_{\sigma * 0}=\frac{1}{3}$ and $T_{\sigma * 1}=0$, or $T_{\sigma * 0}=T_{\sigma * 1}=0$. Then, if $\beta \geq 0, \Phi(\beta)=E\left(T_{0}^{\beta}+T_{1}^{\beta}\right)=p_{\text {both }} \cdot 2 \cdot\left(\frac{1}{3}\right)^{\beta}+$ 
$p_{\text {left }} \cdot\left(\frac{1}{3}\right)^{\beta}+p_{\text {right }} \cdot\left(\frac{1}{3}\right)=\left(2 p_{\text {both }}+p_{\text {left }}+p_{\text {right }}\right)\left(\frac{1}{3}\right)^{\beta}=\left(1+p_{\text {both }}-p_{\text {die }}\right)\left(\frac{1}{3}\right)^{\beta}$, so $\Phi(0)>1 \Longleftrightarrow p_{\text {both }}>p_{\text {die }}$, hence we can assured that $K$, and hence $Q$, is nonempty with positive probability. Also, by Theorem 2 we know that $P\left(\operatorname{dim}_{H}(K)=\log _{3}\left(1+p_{\text {both }}-p_{\text {die }}\right) \mid K \neq \emptyset\right)=1$ and hence by Lemma 5 , we know that $P\left(\operatorname{dim}_{H}(Q)=\log _{3}\left(1+p_{\text {both }}-p_{\text {die }}\right) \cdot d=\log _{2}\left(1+p_{\text {both }}-p_{\text {die }}\right) \mid Q \neq \emptyset\right)=1$, i.e. if $Q$ is nonempty, $\operatorname{dim}_{H}(Q)=\log _{2}\left(1+p_{\text {both }}-p_{\text {die }}\right)$ almost surely. Also by Theorem 3 and Lemma 5, we know that $\mathcal{H}^{\log _{2}\left(1+p_{\text {both }}-p_{\text {die }}\right)}(Q)=0$ almost surely.

Theorem 4 implies that if $g(t)=t^{\log _{3}\left(1+p_{\text {both }}-p_{\text {die }}\right)}\left(\log (|\log (t)|)^{1-\log _{3}\left(1+p_{\text {both }}-p_{\text {die }}\right)}\right.$ $P\left(0<\mathcal{H}^{g}(K)<\infty \mid K \neq \emptyset\right)=1$, and hence by Lemma $6, P\left(0<\mathcal{H}^{h}(Q)<\infty \mid Q \neq\right.$ $\emptyset)=1$.

\section{An Additional General Model}

One can analyze the following general model. Let $P$ be a probability distribution on the sets of all finite trees, $S^{*}$. The process begins by choosing a tree according to $P$, and then for each end node of the tree, independently choose trees according to $P$ and append it to those nodes. This is the specific model outlined in [1], and it could be viewed as a random recursive construction by using $\prod_{i \in \mathbb{N}}\left(S^{*}, P\right)$. Because random recursive fractal constructions can use words from $\mathbb{N}^{*}$, one could view the process of appending a random tree from $S^{*}$ as simply another random recursion, one could define the $J_{\mathrm{S}}$ in a way so that the number of children produced is not done node-by-node, but instead is done tree-by-tree.

\section{REFERENCES}

[1] George Barmpalias; Paul Brodhead; Douglas Cenzer; Seyyed Dashti; Rebecca Weber, Algorithmic Randomness of Closed Sets, Journal of Logic and Computation, Vol. 17, No. 6, pg. 1041-1062 (2007)

[2] Artemi Berlinkov; R. Daniel Mauldin, Journal of Theoretical Probability 15, pg. 695-713 (2002)

[3] Kenneth Falconer, Fractal Geometry: Mathematical Foundations and Applications, John Wiley \& Sons Ltd., Chichester, 1990

[4] Siegfried Graf, Statistically Self-Similar Fractals, Probability Theory and Related Fields 74, pg. 357-392 (1987)

[5] Siegfried Graf; R. Daniel Mauldin; S. C. Williams, Memoirs of the American Mathematical Society Vol. 71, No. 381 (1988)

[6] R. Daniel Mauldin; S. C. Williams, Transactions of the American Mathematical Society, Vol. 295, No. 1, pp. 325-346. (1986)

[7] Pertti Mattila, Geometry of Sets and Measures in Euclidean Spaces: Fractals and rectifiability, Cambridge

Studies in Advanced Mathematics, Vol. 44, Cambridge University Press, 1995

[8] C.A. Rogers, Hausdorff Measures, Cambridge University Press, 1970

Mathematics Department, University of North Texas, P.O. Box 311430, Denton, TX 76203-1430

E-mail address: mauldin@unt.edu,mclinden@unt.edu 